\title{
Agenesis or late maturity of primary canines?
}

\author{
I. Kjaer $^{1} \cdot$ J. Daugaard-Jensen ${ }^{2}$
}

Received: 20 December 2016/ Accepted: 17 February 2017/Published online: 24 February 2017

(C) European Academy of Paediatric Dentistry 2017

The December 2016 issue of European Archives of Paediatric Dentistry contained a short communication (Anthonappa and King 2016) demonstrating the dentition of a 4-year-old Chinese female whose primary canines were unerupted. From the orthopantomogram shown in the article, four canines which were not erupted had approximately a half-sized root length. According to the textbook Paediatric Dentistry-A Clinical Approach by Koch and Poulsen (2009), the average age of a child with a half-sized primary canine is $1 \frac{1 / 2}{2}$ years, while the age of a child with a half-sized permanent canine is approximately 8 years.

It is well known that patients with agenesis in general are late in reaching dental maturity (Daugaard et al. 2010). With this background in mind, we suggest that the four canines in the article presented are primary canines severely late in maturity and that agenesis of the permanent canines has occurred. If this is correct, then primary canine maturity and eruption should be studied as a predictor of permanent canine agenesis.

This short communication forms an excellent basis for future studies on the relationship between maturity of primary dentition and agenesis of permanent teeth.

\section{References}

Anthonappa RP, King NM. Hypodontia of all primary canines. Eur Arch Paediatr Dent. 2016;17(6):485-7. doi:10.1007/s40368-0160259-3.

Daugaard S, Christensen IJ, Kjær I. Delayed dental maturity in dentitions with agenesis of mandibular second premolars. Orthod Craniofac Res. 2010;13:191-6.

Koch G, Poulsen S. Paediatric dentistry-a clinical approach, vol. 2. New York: Wiley; 2009. p. 188.
I. Kjaer

ik@sund.ku.dk

1 Institute of Odontology, Faculty of Health Sciences, University of Copenhagen, Copenhagen, Denmark

2 Department of Oral and Maxillofacial Surgery and Rare Oral Diseases, Rigshospitalet, Copenhagen University Hospital, Copenhagen, Denmark 